\title{
COMPARISON OF CONTROL TECHNIQUES FOR BAKER'S YEAST CULTURE USING AN AUTOMATIC GLUCOSE ANALYZER
}

\author{
KAZUYUKI SHIMIZU, MAKOTO MORIKAWA, SATORU MIZUTANI, \\ SHINJI IIJIMA, MASAKAZU MATSUBARA AND TAKESHI KOBAYASHI \\ Department of Chemical Engineering, Nagoya University, Nagoya 464
}

Key Words: Biochemical Enginecring, Process Control, Fed Batch Culture, Glucose Concentration, Automatic Glucose Analyzer, Baker's Yeast Culture

\begin{abstract}
A comparative evaluation of on-line control techniques was made for the glucose-controlled fed-batch culture of baker's yeast. Based on the comparison of control qualities by computer simulation, two methods -moving identification combined with optimal control and programmed-PI control- were adopted for the experiments to keep the glucose concentration in the culture broth at low level. The control variable was the feed rate of concentrated glucose. The glucose concentration in the culture broth was measured on-line every $\mathbf{5}$ min using an automatic glucose analyzer. The glucose concentration in the broth was controlled in the range of $0.1-0.2 \mathrm{~kg} / \mathrm{m}^{3}$ with these control strategies.
\end{abstract}

\section{Introduction}

Applications of computers to fermentation processes have increased in recent years and many researchers tend to use computers coupled with probes and analyzers. Along with this, various control algorithms have been developed for fermentation processes. ${ }^{6)}$

It is known that in a culture of high sugar concentration, the cellular yield of baker's yeast is depressed due to the Crabtree effect. On the other hand, if the sugar feed is not sufficient, the productivity of cell mass is decreased. Therefore, appropriate sugar feeding is essential to attain high values of cellular yield and productivity. The major problem has been the difficulty in monitoring sugar concentration in the culture broth. Several researchers ${ }^{1,2)}$ used the respiratory quotient (RQ) to determine the appropriate sugar feed rate, while others ${ }^{3,4)}$ used the ethanol concentration as the controlled variable. Although they showed some success, control quality has been limited in the sense that glucose concentration in the culture broth is not tightly maintained at the set value since the measurement variables are indirectly related to the glucose concentration.

In our previous paper, ${ }^{8)}$ we reported the basic characteristics of an automatic glucose analyzer and on-off control of glucose concentration. In the present study, we apply several more sophisticated control techniques to show the impact of using the glucose analyzer to inprove the cellular yield.

The difficulty in designing a control system for

Received May 29, 1987. Correspondence concerning this article should be addressed to T. Kobayashi fermentation processes is due to the lack of accurate mathematical models that describe the dynamics because of the complexity in metabolic reactions and the time-varying inherent nonlinear natures of the system equations, etc. The development of modern control techniques to cope with those problems is still in progress, and it is difficult at this stage to say which of the available techniques is the best for the fed-batch culture of baker's yeast. It is therefore useful to evaluate several promising control techniques for maintaining the glucose concentration in the culture broth tightly at low level.

\section{Description of On-Line Control Techniques}

Before discussing the experimental evaluation, it is reasonable to make a proper comparative evaluation of the available on-line control techniques by computer simulation. In this section, the several control techniques considered are briefly described.

Although three control techniques are discussed in this paper, many others are of course available. Recently, Montague et al. ${ }^{9)}$ applied the generalized predictive control algorithm and the suboptimal LQG (Linear Quadratic Gaussian) algorithm combined with the self-tuning controller to industrial fedbatch penicillin fermentation. Williams et al. ${ }^{16)}$ studied the feasibility of applying multivariable adaptive control to the fed-batch culture of $\mathrm{Sac}$ charomyces cerevisiae. These strategies may be considered as well for a fed-batch culture using glucose analyser.

\subsection{Moving identification combined with optimal con- trol (MICOC)}

The first approach is based on the assumption that 
the process model is not known at all but the time series data of input and output variables are available on-line. The system is linear over a short time period if we have

$$
S_{i}=A S_{i-1}+B F_{i-1}
$$

where $S_{i}$ is the measured glucose concentration in the culture broth at the $i$-th sampling time. $F_{i-1}$ is the nutrient feed rate supposed to be constant during the $(i-1)$ th and the $i$-th sampling times. Since $A$ is the system pole, its absolute value must be less than unity. The assignment of $A$ is rather arbitrary and we set 0.5 in our experimental studies. The assignment of $A$ has little effect on determining moving models. The reason is that the parameter $B$ is adjustable on-line using the least-squares method and $F_{i-1}$ was determined so that optimal control is attained in the sense that the squared error is minimized. ${ }^{17)}$

\subsection{Programmed control}

The second approach is based on the idea that some of the growth parameters may be roughly obtained from the batch data. Then the expected feed rate, $F^{*}(t)$, is computed as a function of time so that the glucose concentration in the broth is kept at the desired level. It can be shown ${ }^{14)}$ that if the substrate concentration in the culture broth is kept roughly constant, the following equation approximately holds:

$$
F^{*}(t)=(\mu / Y+m) X V /\left(S_{F}-S^{*}\right)
$$

where $V, S_{F}$ and $S^{*}$ are fermentor volume, substrate concentration of the feed and set-point value of the substrate concentration, respectively. $Y, \mu$ and $m$ are the yield coefficient, specific growth rate and maintenance coefficient, respectively. The estimated biomass concentration, $X$, can be obtained by the mass balance equations. ${ }^{5,10)}$ Because of the uncertainty in the mathematical model and disturbances influenced by the environmental changes, some sort of feedback compensation is required. ${ }^{14)}$ As the feedback compensator, we considered $\mathrm{P}$ and PI control.

\subsection{Model reference adaptive control}

The third approach is the well-known model reference adaptive control (MRAC) techniques ${ }^{7,14)}$ in which the control parameters are adjusted so as to minimize the difference between the process response and the reference model response.

\section{Experimental}

In the experiments the glucose concentration in the culture broth was monitored on-line every $5 \mathrm{~min}$, using the automatic glucose analyzer as described in our previous paper. ${ }^{8}$ ) The feed rate of the concentrated glucose $\left(200 \mathrm{~kg} / \mathrm{m}^{3}\right)$ was manipulated to control the glucose concentration in the broth at $0.15 \mathrm{~kg} / \mathrm{m}^{3}$ by a programmable peristaltic pump (Atto
Co., Type AC-2120) through the use of a DA converter (Contec Co., DA12-4 (98)) interfaced to a personal computer (NEC Corp., PC-9801). The microorganism used in this study was $S$. cerevisiae. The culture medium and culture conditions were the same as those reported previously. ${ }^{8)}$

Since the glucose electrode cannot be inserted in the fermentor, about $2 \mathrm{~cm}^{3}$ of sample broth was transferred to the glucose analyzer every sampling time. The sample transferred must be microorganism-free, and the microorganism was removed by a cross-flow filtration unit. The detailed scheme around the glucose analyzer has been given previously. ${ }^{8)}$ The problem is the clogging of the filter by the accumulation of the microorganism, which causes a response delay in the glucose analyzer. A membrane filter (Pall Co., pore size: $0.2 \mu \mathrm{m}$, diameter: $7 \mathrm{~cm}$ ) and a ceramic filter (Toshiba Ceramics Co., pore size: $0.5 \mu \mathrm{m}$, length: $11 \mathrm{~cm}, 12$ holes of $4 \mathrm{~mm}$ diameter) were used in the filtration unit. To prevent clogging of the membrane filter by the microorganism, the superficial liquid velocity of the culture broth was set at $30 \mathrm{~cm} / \mathrm{s}$. The ceramic filtration unit was the same as reported by Taniguchi et al. ${ }^{15)}$

\section{Results and Discussion}

\subsection{Comparative evaluation of control techniques}

The simulation result for MICOC showed that although the glucose concentration in the broth was tightly maintained around the set point $\left(0.28 \mathrm{~kg} / \mathrm{m}^{3}\right)$, it tended to fluctuate severely as the sampling interval was increased and as the cell concentration became high. This is because the method is based on the assumption that the system is linear over the range of several sampling intervals used for system identification.

The simulation result for the programmed Pcontrol scheme showed that a large offset tended to accumulate as time goes on because of the model's inaccuracy. This may be overcome by changing the controller gain with respect to time, but it is not an easy task to find the appropriate gain scheduling. Another way to overcome the above difficulty is to incorporate integral action. The simulation result for the programmed PI-control scheme showed that the glucose concentration in the broth was controlled at a satisfactory level without offset when the modeling error in the specific growth rate was within $10 \%$.

For the simulation using the control scheme of MRAC, most of the design parameters were set at the same value as stated in Takamatsu et al. ${ }^{14)}$ Some of the parameters associated with the control law ${ }^{7)}$ were obtained by trial and error so as to give good response. The simulation result shows that some fluctuations of glucose concentration in the broth were observed and that control quality was not good as 
compared with the two methods discussed above. In particular, the IAE (integral absolute error) for MRAC was about twice that for MICOC for $12 \mathrm{~h}$ of fermentation. Because of this, we adopted MICOC and programmed PI-control schemes for the experiments.

\subsection{Preliminary experimental results}

In our previous paper, we could control the glucose concentration in the broth by on-off control using the glucose sensor combined with a ceramic filtration unit. $^{8)}$ Here, we applied more sophisticated control strategies with the filtration system.

Figure 1 shows the experimental results of the fed-batch culture with MICOC and programmed PIcontrol strategies. The dashed line in Fig. 1 indicates that the glucose concentration in the broth was somehow maintained around the set point until about $5 \mathrm{~h}$ while it tended to fluctuate after that time. Finally the system became unstable. The solid line in Fig. 1 shows that the glucose concentration in the broth was maintained roughly around the set point until around $6 \mathrm{~h}$, but the offset for the PI-control tended to accumulate as time proceeded. Before attempting more sophisticated control strategies to overcome the above-mentioned problems, we carefully examined the source of deterioration in control quality.

\subsection{Selection of filtration unit}

Figure 2 shows the step responses of glucose concentration in the culture broth before and after experiments using either the ceramic filter or the membrane filter. As can be seen, the time constant of the ceramic filter changed from 5 to $23 \mathrm{~min}$ as time proceeded because of the accumulation of the microorganism. Unlike the case of the ceramic filter, the response of the membrane filter was found to be quite fast (the time constant was about $3 \mathrm{~min}$ ) even at the late stage of the culture. Since the accumulation of the microorganism was considered to be the main source of deterioration in control quality, we used the membrane filtration unit in the following experiments.

\subsection{Fed-batch culture with on-line control}

Figure 3 shows the result of a fed-batch culture with MICOC technique. Although the glucose concentration sometimes fluctuated, it was maintained between 0.1 and $0.2 \mathrm{~kg} / \mathrm{m}^{3}$ throughout the experiment. Figure 4 shows the corresponding optical density at $570 \mathrm{~nm}\left(\mathrm{OD}_{570}\right)$ and the ethanol concentration. The specific growth rate was about $0.3 \mathrm{~h}^{-1}$ and the final value of $\mathrm{OD}_{570}$ reached 98 . The specific ethanol production rate was $0.02 \mathrm{~kg}$ ethanol $/(\mathrm{kg}$ dry cell $\cdot \mathrm{h})$, which was much smaller than that obtained by on-off control. ${ }^{8}$

Figures 5 and 6 show the results of another fedbatch culture under programmed-PI control. The

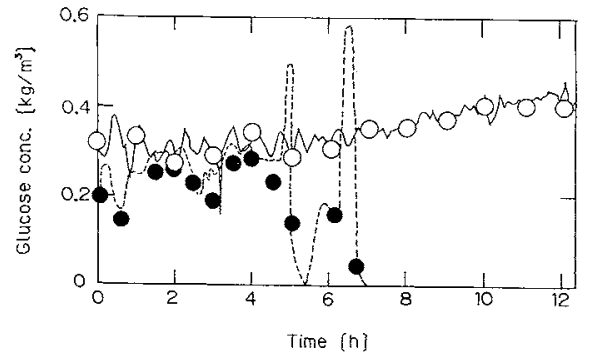

Fig. 1. Change of glucose concentration in fed-batch culture. Ceramic filter was used as a filtration unit. ----, glucose concentration measured on-line with MICOC; glucose concentration determined by off-line measurement for MICOC; - - , glucose concentration measured on-line with programmed PI-control; $O$, glucose concentration determined by off-line measurement for programmed PIcontrol

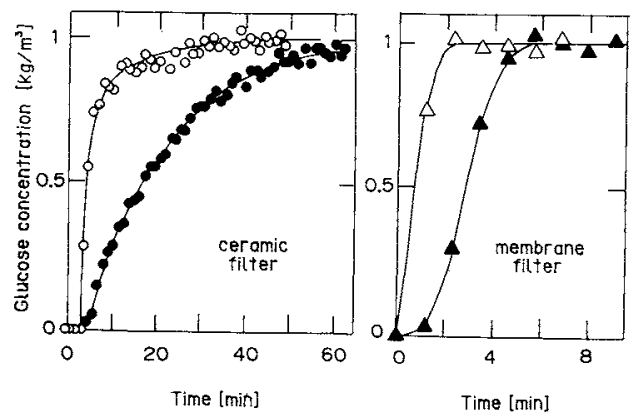

Fig. 2. Step responses of glucose concentration in ceramic and membrane filtration units. $(O)$, before fermentation using ceramic filter; (O), after fermentation using ceramic filter; $(\triangle)$, before fermentation using membrane filter; $(\boldsymbol{\Delta})$, after fermentation using membrane filter

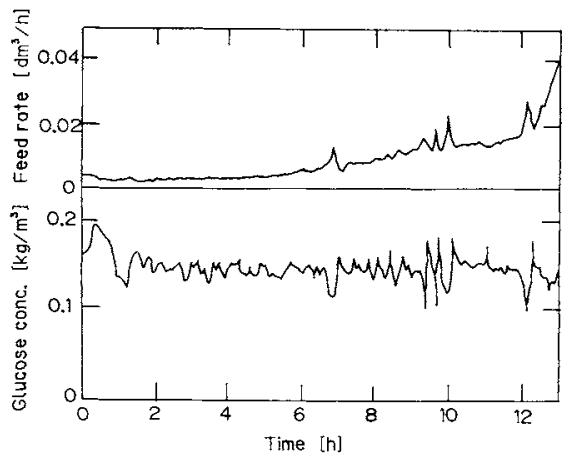

Fig. 3. Time courses of glucose concentration and feed rate in fed-batch culture with MICOC

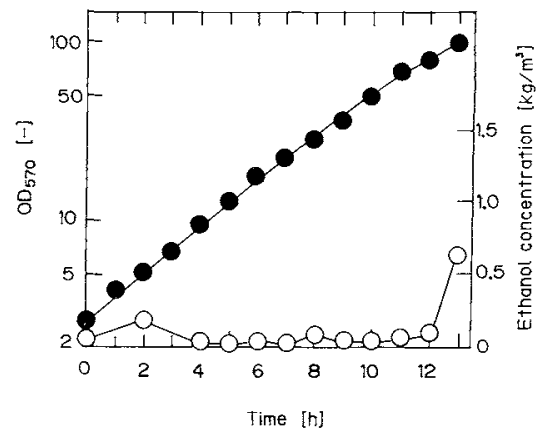

Fig. 4. Time courses of $\mathrm{OD}_{570}(\bigcirc)$ and ethanol concentration $(O)$ in fed-batch culture with MICOC 


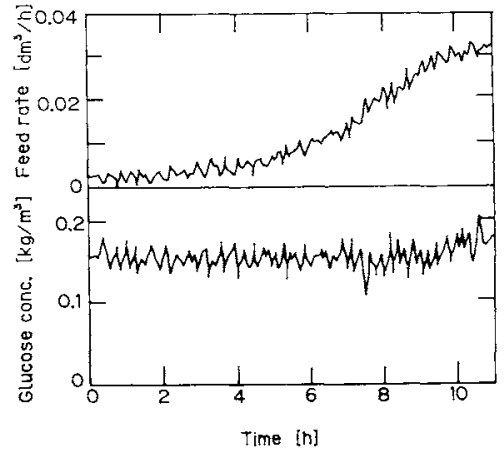

Fig. 5. Time courses of glucose concentration and feed rate in fed-batch culture with programmed-PI control

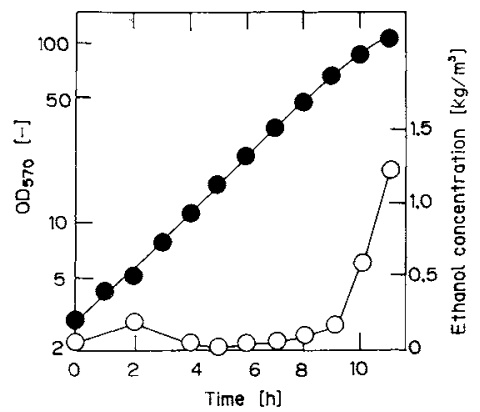

Fig. 6. Time courses of $\mathrm{OD}_{570}(-)$ and ethanol concentration $(O)$ in fed-batch culture with programmed-PI control

specific growth rate was $0.35 \mathrm{~h}^{-1}$ and the final value of $\mathrm{OD}_{570}$ reached 106. The fluctuation of the glucose concentration was small, ranging from 0.13 to $0.2 \mathrm{~kg} / \mathrm{m}^{3}$, although a little offset was observed after about $9 \mathrm{~h}$. The ethanol concentration was kept low until $9 \mathrm{~h}$. However, it increased sharply after $\mathrm{OD}_{570}$ became 80 . Okada et al. ${ }^{10)}$ showed that baker's yeast could grow without producing ethanol if the glucose concentration in the broth was kept below $0.28 \mathrm{~kg} /$ $\mathrm{m}^{3}$. Ogawa et al. ${ }^{11)}$ reported that ethanol was produced by the shortage of phosphate in baker's yeast culture. The sharp increase in ethanol concentration may be due to the shortage of phosphate in the broth because phosphate was not fed and the glucose concentration was kept less than $0.2 \mathrm{~kg} / \mathrm{m}^{3}$. The final ethanol concentration and the final ethanol production rate were $1.23 \mathrm{~kg} / \mathrm{m}^{3}$ and $0.06 \mathrm{~kg}$ ethanol $/ \mathrm{kg}$ dry cell $\cdot h$ ) respectively. Note, however, that the final ethanol concentration per $\mathrm{OD}_{570}$ was one-fourth that obtained by on-off control. ${ }^{8)}$

The offset observed in the late stage of the culture might have been produced by the change of control parameters as time proceeded. If the cell concentration is monitored on-line, a more accurate value for $F(t)$ is obtained using the updated cell concentration.

If the glucose concentration in the culture broth cannot be measured on-line, it must be estimated from the available measurement variables. It is known ${ }^{12,13)}$ that the extended Kalman filter is effec- tive for the estimation of state variables and the parameters of nonlinear systems such as the fed-batch culture system. However, simulation results (data not shown), showed that control quality evaluated based on such criteria as IAE was limited when using indirect measurement, whatever the efficient filter was adopted.

For the particular system of baker's yeast culture, the RQ or the ethanol concentration can be used as well for the measurement variable. However, the significance of using an automatic glucose analyzer is that it can be applied not only to the baker's yeast culture but also to other types of cultures for efficient utilization of glucose and production of metabolites. An example of a culture of Escherichia coli harboring a recombinant plasmid will be published elsewhere.

\section{Conclusion}

The results of both computer simulation and experiment indicated that satisfactory control could be achieved to obtain high cellular yield by the on-line measurement of glucose concentration using either moving identification combined with optimal control or programmed-PI control strategy to keep the glucose concentration in baker's yeast culture at a low level of around $0.1-0.2 \mathrm{~kg} / \mathrm{m}^{3}$.

\section{Acknowledgments}

The authors are grateful to Mr. Rokuro Izumi, Toyo Jozo Co., Ltd., for invaluable discussion. This work was supported in part by a Grant-in-Aid for General Project (No. 62470112) from the Ministry of Education, Science and Culture, Japan.

$\begin{array}{llr}\text { Nomenclature } & \\ A & =\text { model parameter for MICOC } & {[-]} \\ B & =\text { model parameter for MICOC } & {[-]} \\ F & =\text { feed flow rate } & {\left[\mathrm{m}^{3} / \mathrm{h}\right]} \\ F^{*} & =\text { expected feed flow rate to keep } S \text { at } S^{*} & {\left[\mathrm{~m}^{3} / \mathrm{h}\right]} \\ m & =\text { maintenance coefficient } & {\left[\mathrm{h}^{-1}\right]} \\ \mathrm{OD}_{570} & =\text { optical density at } 570 \mathrm{~nm} & {[-]} \\ S & =\text { substrate concentration } & {\left[\mathrm{kg} / \mathrm{m}^{3}\right]} \\ S^{*} & =\text { set-point value for substrate } & {\left[\mathrm{kg} / \mathrm{m}^{3}\right]} \\ & & {\left[\mathrm{kg} / \mathrm{m}^{3}\right]} \\ S_{F} & =\text { feed substrate concentration } & {\left[\mathrm{m}^{3}\right]} \\ V & =\text { fermentor volume } & {\left[\mathrm{kg} / \mathrm{m}^{3}\right]} \\ X & =\text { cell concentration in broth } & {[-]} \\ Y & =\text { yield coefficient } & \\ & & \end{array}$

\section{Literature Cited}

1) Aiba, S., S. Nagai and T. Nishizawa: Biotechnol. Bioeng., 18, 1001 (1976).

2) Cooney, C. L., H. Y. Wang and D. I. C. Wang: Biotechnol. Bioeng., 19, 55 (1977).

3) Dairaku, K., Y. Yamasaki, K. Kuki, S. Shioya and T. Takamatsu: Biotechnol. Bioeng., 23, 2069 (1981).

4) Dairaku, K., E. Izumoto, H. Morikawa, S. Shioya and T. Takamatsu: J. Ferment. Technol., 61, 189 (1983).

5) Fukuda, H., T. Shiotani, W. Okada and H. Morikawa: $J$. 
Ferment. Technol, 56, 361 (1978).

6) Johnson A. (ed.): "Proc. of the Symposium on Modelling and Control of Biotechnological Processes," Noordwijkerhout, the Netherlands (1986).

7) Lozano, R. and I. D. Landau: Int. J. Control, 33, 247 (1981).

8) Mizutani, S., S. Ijima, M. Morikawa, K. Shimizu, M. Matsubara, Y. Ogawa, R. Izumi, K. Matsumoto and T. Kobayashi: J. Ferment. Technol., 65, 325 (1987).

9) Montague, G. A., A. J. Morris, A. R. Wright, M. Aynsley and A. Ward: Can. J. Chem. Eng., 64, 567 (1986).

10) Okada, W., H. Fukuda and H. Morikawa: J. Ferment. Technol., 59, 103 (1981).

11) Ogawa, T., T. Inoue, S. Sato, M. Shimoda and S. Aiba:
Biotechnol. Lett., 2, 147 (1980).

12) Stephanopoulos, G. and K.-Y. San: Biotechnol. Bioeng., 26, 1176 (1984).

13) Svrcek, W. Y., R. F. Elliot and J. E. Zajic: Biotechnol. Bioeng., 16, 827 (1974).

14) Takamatsu, T., S. Shioya, Y. Okada and M. Kanda: Biotechnol. Bioeng., 27, 1675 (1985).

15) Taniguchi, M., N. Kotani and T. Kobayashi: J. Ferment. Technol, 65, 179 (1987).

16) Williams, D., P. Yousefpour and E. M. H. Wellington: Biotechnol. Bioeng., 28, 631 (1986).

17) Wu, W.-T., K.-C. Chen and H.-W. Chiou: Biotechnol. Bioeng., 27, 756 (1985).

\title{
EFFECT OF IMMOBILIZATION ON STABILITY OF RECOMBINANT PLASMIDS IN GENE-ENGINEERED MICROORGANISMS
}

\author{
OSAMU ARIGA, TOHRU WATARI, KIMIHISA TAKEUCHI, \\ MASATAKA ANDO, HIROMI TAKAGI AND YOSHIKI SANO \\ Department of Fine Materials Engineering, Shinshu University, Nagano 386 \\ SHINJI IIJIMA AND TAKESHI KOBAYASHI \\ Department of Chemical Engineering, Nagoya University, Nagoya 464
}

\begin{abstract}
Key Words: Biochemical Engineering, Stability of Plasmid, Immobilized Cells, Gene Engineering, trp Promoter

Stability of a recombinant plasmid pMCT98 was investigated for free and immobilized Escherichia coli C600/pMCT98 cells. KCl, a hardening reagent for immobilizing matrix, inhibited cell growth and decreased the stability of the plasmid for free cells. However, the immobilization within $\kappa$-carrageenan gel did not affect cell growth and increased significantly the stability of the plasmid, and the amount of the gene product did not decrease through successive passage of the immobilized cells. Coexistence of plasmid pRLK13 lowered the stability of plasmid pMCT98 for both free and immobilized cells. Some oscillations in the ratio of cells harboring the plasmids were observed in the immobilized $E$, coli C600/pMCT98/pRLK13 system.
\end{abstract}

\section{Introduction}

For production of useful materials by recombinant DNA technology, the target gene is introduced into a vector plasmid and the productivity of gene products may be enhanced by gene dosage effect. Plasmid loss, in turn, results in decreasing productivity. Because great numbers of generations are required for largescale culture, the stability of recombinant plasmid becomes important. Plasmid stability has been investigated by many workers. ${ }^{3,10,13,16)}$ Jones et al. ${ }^{10)}$ reported that plasmid-free segregants were observed in the case of recombinant Escherichia coli after 30 generations under chemostat culture without selective pressure such as antibiotics, in which phosphate and glucose were limited. By contrast, Noack et al. ${ }^{16)}$

\footnotetext{
Received June 4, 1987. Correspondence concerning this article should be addressed to
} Y. Sano. reported that plasmid pBR322 was extremely stable in antibiotic-free chemostat under both glucose and ammonium limitations. Efforts to stabilize plasmid by genetic methods have also been studied by many investigators. Skogman et al. ${ }^{18)}$ used the partition locus to enhance the stability of trp-operon bearing plasmid. Yukawa et al. ${ }^{23)}$ stabilized a pBR322 derivative by using the mini-F fragment.

When gene products are industrially produced, immobilization techniques are very attractive. For instance, ethanol production using immobilized yeast has been extensively investigated. ${ }^{5,7,21)}$ However, only a few papers on immobilization of genetically engineered microorganisms have appeared. Inloes et al. ${ }^{9)}$ investigated protein synthesis by $E$. coli harboring a recombinant plasmid using a hollow-fiber membrane reactor. Georgiou et $a l^{4)}$ and Dhulster et al. ${ }^{2)}$ entrapped genetically engineered $E$. coli within a poly- 